\title{
Avaliação do bundle de uso de cateter vesical de demora
}

\author{
Evaluation of the bundle of use of a long deduring bladder catheter \\ Evaluación del haz de uso de un catéter vesical de larga duración
}

Isabelle Fernandes Borsato

ORCID: https://orcid.org/0000-0001-8798-2489 Universidade Federal do Estado do Rio de Janeiro, Brasil E-mail:mbisaborsato@gmail.com

Emanuel Pereira dos Santos

ORCID: https//orcid.org/0000-0003-2454-7572 Universidade Federal do Estado do Rio de Janeiro, Brasil E-mail:emanuelgranarcanjo@gmail.com

Vera Lúcia Freitas

ORCID: https://orcid.org/0000-0003-1324-5640 Universidade Federal do Estado do Rio de Janeiro, Brasil E-mail: veralfreit@gmail.com

Bianca de Oliveira Fonseca

ORCID: https://orcid.org/0000-0002-6251-4977

Universidade do Estado do Rio de Janeiro, Brasil

E-mail: bianca.micro@gmail.com

Gicélia Lombardo Pereira

ORCID: https://orcid.org/0000-0002-4032-2093 Universidade Federal do Estado do Rio de Janeiro, Brasil E-mail:gilombardo@hotmail.com

Beatriz Gerbassi Costa Aguiar

ORCID: https://orcid.org/0000-0001-6815-4354 Universidade Federal do Estado do Rio de Janeiro, Brasil E-mail: beatriz.costa @unirio.br

Rhuani de Cássia Mendes Maciel

ORCID: https://orcid.org/0000-0003-0393-3482 Universidade Federal do Estado do Rio de janeiro, Brasil E-mail:rhuanimaciel@hotmail.com

Júlya de Araujo Silva Monteiro

ORCID: https://orcid.org/0000-0001-6065-5744 Universidade Federal do Estado do Rio de Janeiro, Brasil E-mail: jmonteiro3000@gmail.com

Aquiene Santos da Silva Pires da Costa

ORCID: https//orcid.org/0000-0002-3496-0590 Universidade Federal do Estado do Rio de Janeiro, Brasil Email:aquienepirez@gmail.com

\begin{abstract}
Resumo
Trata-se de um estudo documental que foi realizado no período entre os meses de março a dezembro de 2019, em um Hospital Federal localizado no município do Rio de Janeiro. Os dados foram coletados nos documentos oficiais arquivados no setor de Comissão de Controle de Infecções Hospitalar do hospital em estudo. O estudo tem como objetivo: Avaliar os indicadores de adequação e inadequações dos cateteres vesicais de demora em um Hospital Federal na cidade do Rio de Janeiro. Como objetivo: Avaliar os indicadores de adequação e inadequações dos cateteres vesicais de demora em um Hospital Federal na cidade do Rio de Janeiro. A análise dos dados mostrou inadequação a fixação do cateter e, em relação a manutenção do coletor abaixo do nível do paciente longe do piso, e capacidade da bolsa coletora menor que 2/3. Temos como conclusão: Os dados mostram a importância de reflexão para o cuidado de enfermagem, a observação das normas e orientações para controle de infecções, qualidade de assistência e segurança dos pacientes.
\end{abstract}

Palavras-chave: Cuidado de Enfermagem; Cateteres de Demora; Pacotes de assistência ao paciente.

\section{Abstract}

This is a documentary study that was carried out from March to December 2019, at a Federal Hospital located in the city of Rio de Janeiro. The data were collected in official documents filed with the Hospital Infection Control Commission sector of the hospital under study. The study aims to: Assess the indicators of adequacy and inadequacy of bladder catheters of delay in a Federal Hospital in the city of Rio de Janeiro. Our objective: Assess the indicators of adequacy and inadequacy of bladder catheters of delay in a Federal Hospital in the city of Rio de Janeiro. Data 
analysis showed inadequate catheter fixation and, in relation to keeping the collector below the level of the patient away from the floor, and the capacity of the collection bag less than $2 / 3$. We conclude: The data show the importance of reflection for nursing care, the observation of norms and guidelines for infection control, quality of care and patient safety.

Keywords: Nursing care; Catheters, Indwelling; Patient care bundles.

\section{Resumen}

Se trata de un estudio documental que se realizó de marzo a diciembre de 2019, en un Hospital Federal ubicado en la ciudad de Rio de Janeiro. Los datos fueron recogidos en documentos oficiales archivados en el sector de la Comisión de Control de Infecciones Hospitalarias del hospital en estudio. El estudio tiene como objetivo: Evaluar los indicadores de adecuación e insuficiencia de catéteres vesicales de retraso en un Hospital Federal de la ciudad de Rio de Janeiro. Como objetivo: Evaluar los indicadores de adecuación e inadecuación de los catéteres vesicales de retraso en un Hospital Federal de la ciudad de Rio de Janeiro. El análisis de los datos mostró una fijación inadecuada del catéter y, en relación a mantener el colector por debajo del nivel del paciente alejado del suelo, y la capacidad de la bolsa colectora menos de 2/3. Concluimos: Los datos muestran la importancia de la reflexión para el cuidado de enfermería, la observación de normas y lineamientos para el control de infecciones, la calidad de la atención y la seguridad del paciente.

Palabras clave: Atención de enfermería; Catéteres de permanencia; Paquetes de atención al paciente.

\section{Introdução}

A sondagem vesical de demora é amplamente usada no meio hospitalar para a drenagem de urina para o meio externo de forma não fisiológica. O cateterismo pode ser utilizado de forma contínua e intermitente com o objetivo de realizar esvaziamento e descompressão da bexiga (Nogueira, et al., 2017)

O procedimento de cateterismo vesical de demora é considerado uma técnica invasiva com inserção de uma sonda através da uretra. Desta forma ocorre a invasão de uma área que é considerada estéril, gerando assim uma probabilidade do indivíduo desenvolver infecção do trato urinário (Cavalcante, Braquehais \& Bezerra, 2017)

A partir do momento em que ocorre a invasão do meio estéril pensamos nas possibilidades de uma colonização e infecção da área. Sendo assim, é necessário a elaboração de estratégias para minimizar este tipo de evento adverso. Para obtermos êxito é necessário que haja uma boa atuação profissional usando como ferramenta a vigilância de processos (Nogueira, et al., 2017).

Para realizarmos a vigilância epidemiológica, utilizamos os Bundles que são criados e embasados em literatura científica e vem a fim de estabelecer conjuntos de medidas e ações que devem ser realizadas com objetivo de sistematizar técnicas corretas e melhoras a qualidade de assistência de enfermagem visando como resultado final evitar a infecções de vias urinaria por meio de cateter vesical de demora (Mota \& Oliveira, 2019a).

O Bundle do cateter vesical é estruturado da seguinte forma: técnicas assépticas, tempo de inserção da sonda, indicação clínica, esvaziamento da bolsa coletora quando atingir o nível recomendado e fixação correta da mesma para ambos os sexos (Mota \& Oliveira, 2019a).

Através da vigilância de processos torna-se possível a construção de indicadores em saúde, possibilitando a visualização das ações realizadas no meio hospitalar e cuidados empregados na manutenção do cateter vesical de demora (Mota \& Oliveira, 2019b).

A construção e instalação de bundles irá refletir de forma pratica na assistência. Quando o mesmo é aplicado de forma sistematizada resulta impactos na assistência, através da diminuição de riscos associados a ITU associado ao uso do cateter vesical de demora (Tolentino, et al., 2017)

Segundo Vidal e Gaite, 2018 cerca de 70\% das infecções hospitalares estão associadas a manutenção e manipulação do cateter. $\mathrm{O}$ manejo inadequado do cateter vesical traz prejuízos clínicos ao paciente, podendo gerar no mesmo bacteremias, prostatite, infecção na coluna vertebral. 
Partindo da minha vivência enquanto enfermeira residente da instituição, percebi que havia um alto índice de pacientes com cateter vesical de demora e que na maioria dos casos o Bundle de manutenção do cateter vesical utilizado na instituição não estava sendo cumprido rigorosamente, deixando assim falhas no processo de cuidar.

A partir desta minha percepção empírica da realidade da instituição interessei-me a buscar e solidificar estes dados e trazê-los de forma científica a comunidade, para que a partir desses dados sejam realizadas intervenções necessárias para a melhoria das práticas em saúde relacionada ao cateterismo vesical de demora.

Neste trabalho, o Bundle de manutenção do catete vesical será composto por 3 quesitos: Esvaziamento correto da bolsa, fixação correta de acordo com sexo e posicionamento abaixo do nível do paciente. Através dele, poderemos avaliar se os critérios acima são considerados adequados ou inadequados.

Esse estudo tem como objeto: Os índices de adequação e inadequação dos cateterismos vesicais de demora de um Hospital Federal na cidade do Rio de Janeiro. Sendo elaborada uma questão norteadora: Quais são os índices de adequação e inadequação de cateter vesical de demora em um Hospital Federal na cidade do Rio de Janeiro?

Formulado o objetivo do estudo: Avaliar os indicadores de adequação e inadequações dos cateteres vesicais de demora em um Hospital Federal na cidade do Rio de Janeiro.

\section{Metodologia}

Trata-se de um estudo documental, retrospectivo, abordagem quantitativa, No estudo retrospectivo avalia-se dados e informações colhidas no passado e finalmente para quantificar os dados colhidos, adota-se a abordagem quantitativa, onde aplica-se formulas com um rigor metodológico para quantificar os dados numéricos encontrados na fase de coleta de dados (Fontelles, et. al., 2009).

Segundo Pereira, et al, 2018 nos métodos quantitativos, faz-se a coleta de dados quantitativos ou numéricos por meio do uso de medições de grandezas e obtém-se por meio da metrologia, números com suas respectivas unidades. Estes métodos geram conjuntos ou massas de dados que podem ser analisados por meio de técnicas matemáticas como é o caso das porcentagens, estatísticas e probabilidades, métodos numéricos, métodos analíticos e geração de equações e/ou fórmulas matemáticas aplicáveis a algum processo.

Os dados para o estudo foram coletados em documentos oficiais disponíveis na Comissão de Controle de Infecção Hospitalar-CCIH do hospital provenientes de um formulário que foi aplicado pelos enfermeiros residentes aos pacientes internados na enfermaria de clínica médica do hospital em estudo que consta de 50 leitos, no período de março a dezembro de 2019.

O documento para coleta de dados foi criado a partir do formulário utilizado pela equipe de CCIH (anexo I) do Hospital. Neste formulário contém itens que avaliaram os critérios de adequação e inadequação dos cateteres vesicais de demora incluindo os seguintes itens: Cateter vesical fixado adequadamente, coletor abaixo do nível do paciente e sem encostar no piso, descarte da diurese quando o coletor atingir $2 / 3$ da sua capacidade.

A partir das observações feitas ele é classificado como adequado e inadequado seguindo um bundle de inserção de cateter vesical (ANVISA, 2017).

Posteriormente os dados obtidos serão apresentados em gráficos e tabelas elaboradas no Excel.

O estudo cumpriu as determinações da Resolução CNS 466/2012. O risco da pesquisa se refere à possibilidade remota de identificação do indivíduo ao qual o documento será analisado. Esse risco é sanado pelo documento não conter área de identificação do paciente, impossibilitando assim, a identificação do indivíduo.

Os benefícios se referem a trazer a comunidade científica, dados relevantes, sobre cateter vesical de demora e infecções hospitalares relacionadas à assistência a saúde. 


\section{Resultados e Discussão}

Os dados foram coletados entre os meses de março a dezembro de 2019 sendo analisados 159 documentos.

Gráfico 1. Distribuição dos registros dos dados segundo o mês pesquisado, no período de março a dezembro de 2019 em um Hospital Federal no município do Rio de Janeiro.

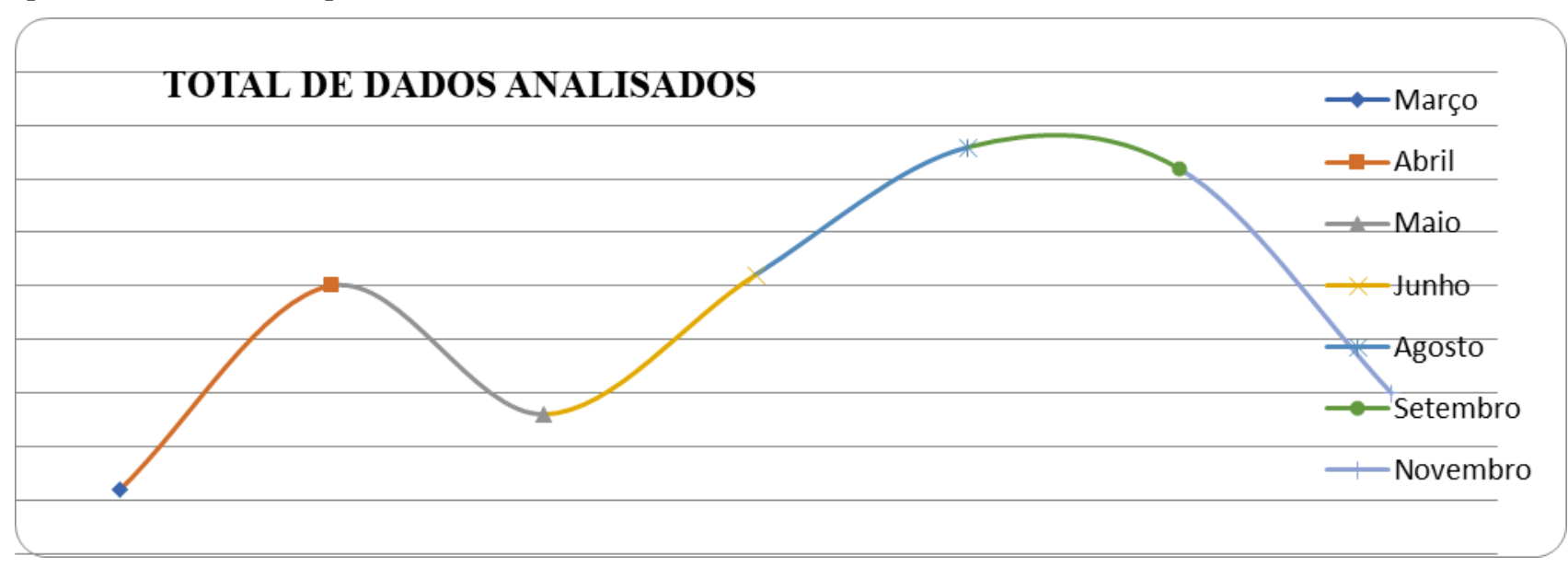

Fonte: Dados do estudo.

Nos meses de julho e dezembro não foram disponibilizados os dados. O Gráfico 1 demonstra visualmente a quantidade de documentos coletados durante os meses, sendo o maior período de coletas dos 159 documentos os meses de agosto e setembro respectivamente. Os menores registros de documentos foram nos meses de março, maio e novembro. A onda mostra a disponibilidade com que a informação foi encontrada durante o período estudado.

Gráfico 2. Fixação do cateter segundo adequação e inadequação analisados segundo o instrumento do Bundle, realizado no período de março a dezembro de 2019 em um Hospital Federal no município do Rio de Janeiro.

\section{FIXAÇÃO DE CATETER}

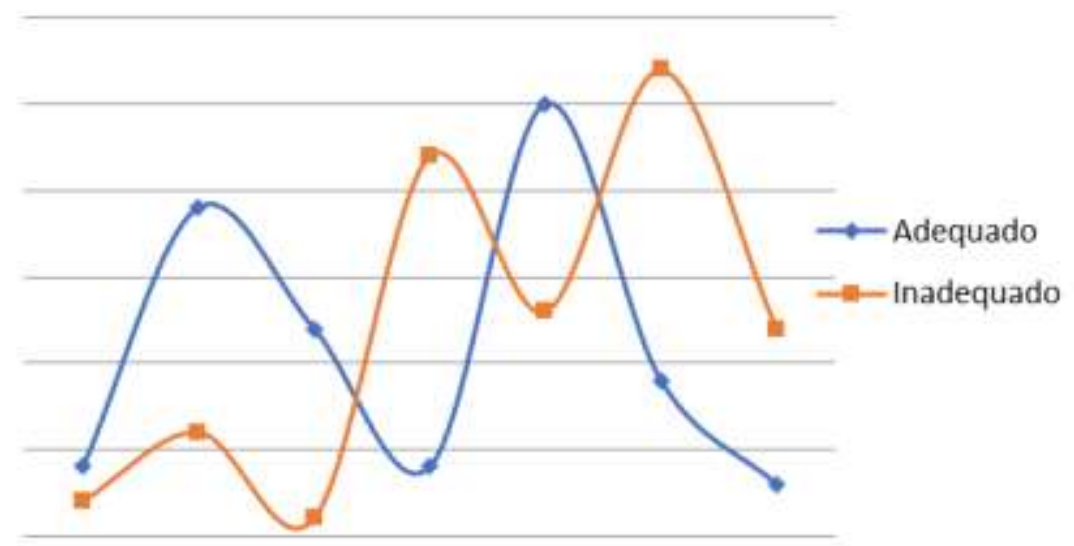

Fonte: Dados do estudo. 
A fixação do cateter obteve índices de inadequação dentro dos dados coletados na instituição. O gráfico apresenta registros de constante fixação inadequada do cateter, apresentando uma oscilação crescente conforme os meses de seu registro. O dado vem a mostrar o contraste entre a fixação adequada com a fixação inadequada do cateter vesical de demora.

A alta inadequação presente nos dados traz a realidade hospitalar e com problemas reais aos pacientes submetidos à técnica de cateterismo vesical de demora. A falta de fixação ou fixação incorreta da sonda gerar microlesões uretrais e sangramento da mesma trazendo facilidade a contaminação.

Segundo Cornistein, et a.l, 2018 as principais vias de contaminação são provenientes da região anal e perianal devido a proximidade anatômica com a região uretral. Além disso, a falta de fixação poderá gerar dor e sangramento do mesmo, caso haja a mobilização e manipulação do paciente (Arrais, et al.,2017).

Levar em consideração o sexo do paciente também é de suma importância, pois a fixação deve ser realizada de acordo com o sexo do mesmo, seguindo a estrutura anatômica do órgão genital de cada sexo (Truzzi, et al.,2017)

A fixação quando o paciente é do sexo feminino dever ser feita na parte interna da coxa e homens na região hipogástrica. Este posicionamento no sexo masculino algumas vezes gera dúvidas e conflitos na equipe. A fixação em região suprapúbica em homens é justificada em literatura para diminuir a tensão entre a região do pênis e saco escrotal e também por homens ter ereções involuntárias, justificadas por picos de liberação hormonal ao longo do dia (Truzzi, et al.,2017)

Gráfico 3. Coletor abaixo do nível do paciente longe do chão segundo os dados avaliados no período de março a dezembro de 2019 em um Hospital Federal no município do Rio de Janeiro.

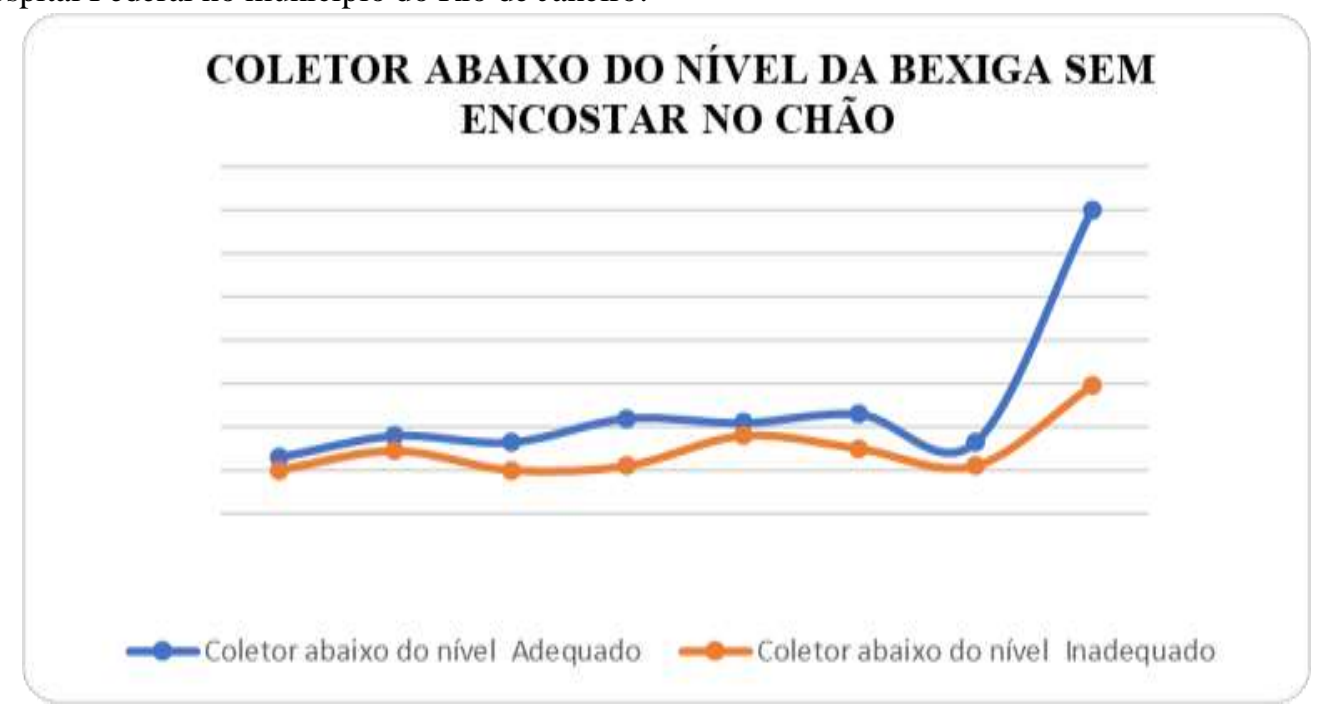

Fonte: Dados do estudo.

Os dados do Gráfico 3 mostram uma taxa de adequação da manutenção do coletor abaixo do nível do paciente sem encostar-se no piso é maior do que a taxa de inadequação. Isso é um ótimo resultado quando pensamos em pacientes com cateterismo vesical de demora, pois isso significa menores probabilidades de eventos adversos aos pacientes principalmente aquelas relacionadas a infecções de vias urinárias por dispositivos.

O coletor abaixo do nível do paciente significa que diminui significativamente as chances de refluxo de urina do paciente podendo carrear microorganismo para o meio estéril (Chaves \& Moraes, 2015).

Os microorganismos mais associados ITU relacionados ao cateter vesical são E. Fecalis, Enterecocus Faeclum, Staphylococus Epidermides (8,53). Pacientes que apresentem sintomas como febre maior que 38 graus, leucocitose ou leucopenia, incontinência urinária e piúria deverão ter a atenção redobrada pois poderá ser um possível indicador de infecção associada ao uso do cateter vesical de demora (Artero, et al., 2018) 
Gráfico 4. Descarte da diurese menor que $2 / 3$ da bolsa registrada nos documentos analisados no período de março a dezembro de 2020 em um Hospital Federal no município do Rio de Janeiro.

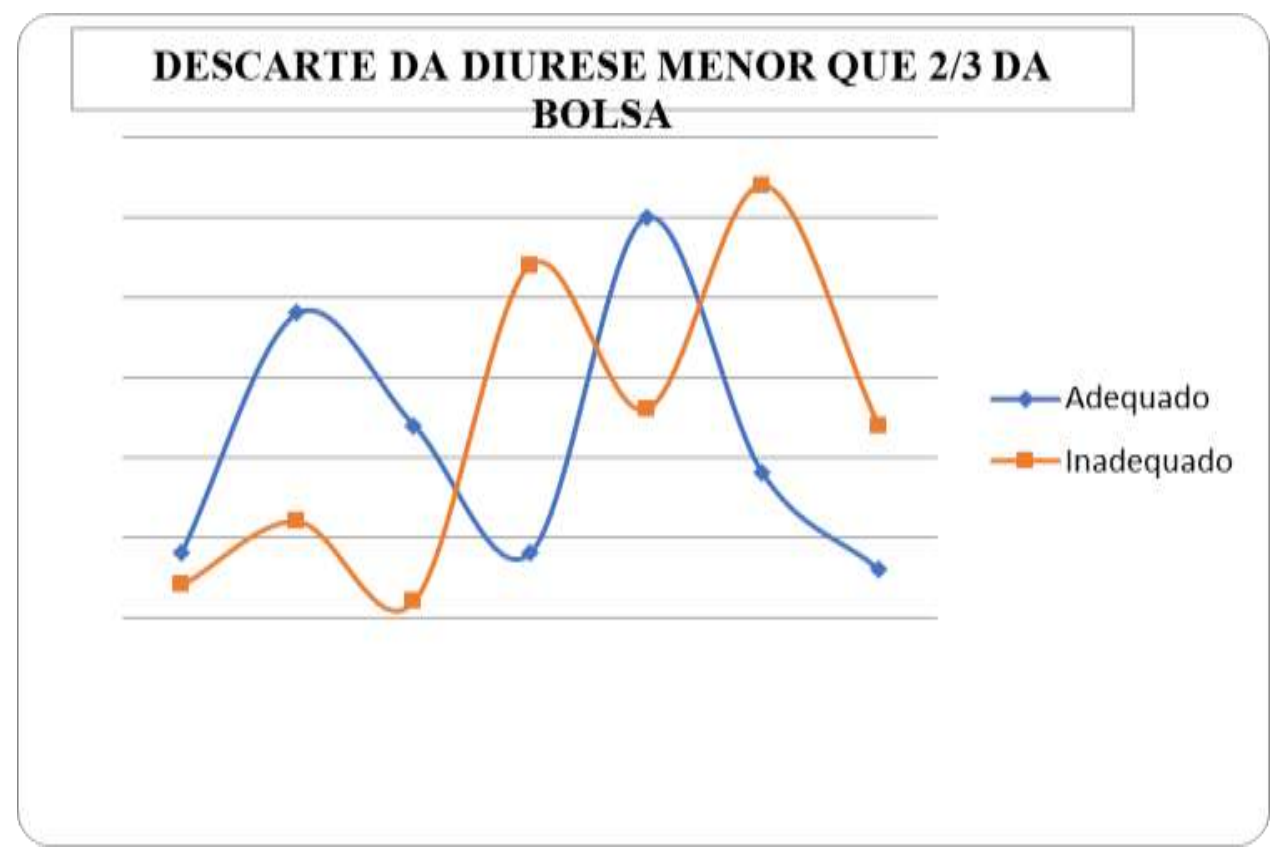

Fonte: Dados do estudo.

Ao analisarmos o descarte da diurese percebe-se uma oscilação entre procedimento realizado de forma adequada e inadequada, porém a inadequação do procedimento vem em destaque ao longo dos meses. A oscilação crescente da inadequação do descarte da diurese na bolsa coletora vem se acumulando durante os meses sinalizando maior risco de lesão e de infecção.

O descartar a urina quando a mesma atinge 2/3 da bolsa evita o refluxo da urina remanescente da bolsa para bexiga, além disso, o peso da urina poderá causar microlesões na uretra deste paciente. O acúmulo de urina na bolsa coletora poderá se tornar meio de proliferação bacteriana. (Chaves \& Moraes, 2015).

A proliferação bacteriana poderá trazer consequências aos pacientes, principalmente aqueles que possuem longo tempo de internação, idade avançado (maior que 60 anos), uso de corticosteróides, uso de aminas e diabetes mellitus (Miranda, et al., 2018)

A contaminação do cateter vesical de demora ocorre de 2 formas: contaminação intra luminal e extra luminal. A forma intra luminal geralmente a contaminação ocorre a passagem da sonda, já a extra luminal ocorre a pós a passagem da sonda, no momento da manutenção do cateter. Neste caso a utilização do BUNDLE se torna uma ferramenta fundamental para que haja a prevenção deste tipo de colonização (Silva, et al., 2019).

\section{Conclusão}

O estudo mostra os cuidados registrados sobre o uso de cateter de demora em pacientes internados. O uso do Bundle para análise dos dados foi possível observar a adequação e inadequação de utilização do cateter, sugerindo a possibilidade de eventos adversos ao paciente.

Os dados mostram a importância de reflexão para o cuidado de enfermagem, a observação das normas e orientações para controle de infecções, qualidade de assistência e segurança dos pacientes. 
Como sugestão, o referido estudo poderá ser replicado em outras unidades de internação e na mesma onde foi realizado para verificar como estão sendo respeitados normas e cuidados para uma boa qualidade de assistência e observar possíveis melhoras na unidade estudada durante o tempo.

\section{Referências}

Artero, A. E., Nuneza, C., Calvo, C. O., Garcia, B. M., \& Liedias, P. (2019). Infección urinaria en el anciano. Elsevier Espa na, S.L.U. y Sociedad Espa nola de Medicina Interna (SEMI). 219(4):189-193. Doi:10.1016/j.rce.2018.10.009

Brasil. Agência Nacional de Vigilância Sanitária Medidas de Prevenção de Infecção Relacionada à Assistência à Saúde. Brasília: Anvisa, (2017). https://www20.anvisa.gov.br/segurancadopaciente/index.php/publicacoes/item/caderno-5

Cavalcante, T. D. M. C., Braquehais, A. R., \& Bezerra, P. D. A. R. G. (2017). Sonda Vesical de Demora: perfil epidemiológico da Infecção Urinária no Centro de Terapia Intensiva RETEP. Rev. Tendên. da Enferm. Profis.; 9(2),2164-2169. 10.5935/1415-2762.20160043

Chaves, N. M. D., \& Moraes C. L. K. (2015). Controle de infecção em cateterismo vesical de demora em unidade de terapia intensiva. R. Enferm. Cent. $o$. $\min , 5(2), 1650-1657$

Cornistein, W. et al. (2018). Infección del tracto urinário asociada a sonda vesical. actualización y recomendaciones intersociedades. Medicina (buenos aires). (78) 258-264. https://www.medicinabuenosaires.com/indices-de-2010-a-2018/volumen-78-ano-2018-no-4-indice/infeccion-del-tracto-urinario-asociada-asonda-vesical-actualizacion-y-recomendaciones-intersociedades/

Fontanelles, M. J., Simões M. G., Farias, S. H., \& Fontenelles, R. G. S. (2009). Metodologia da pesquisa científica: diretrizes para a elaboração de um protocolo de pesquisa. Scientific research methodology: Guidelines for elaboration of a research protocol. Revista Paraense de Medicina. https://rsdjournal.org/index.php/rsd/article/view/11724/10457

Miranda, L. A et al. (2016). Results after implementation of a protocol on the incidence of urinary tract infection in an intensive care unit. Rev. Latino-Am. Enfermagem. (24)2-9. 10.1590/1518-8345.0866.2804

Mota E. C., \& Oliveira A. C. (2019 b). Prevenção de infecção do trato urinário associada a cateter: qual o gap na prática clínica? Texto Contexto Enferm. 12(8),1-12. 10.1590/1980-265x-tce-2018-0050

Mota, E. C., \& Oliveira A. C (2019a). Catheter-associated urinary tract infection: why do not we control this adverse event? Rev Esc Enferm USP. 53. $10.1590 / \mathrm{s} 1980-220 \times 2018007503452$

Nogueira, H. K. L., et al. (2017) Conhecimento de profissionais intensivistas sobre o bundle para a prevenção de infecção do trato urinário associada ao uso de sondas. Rev enferm UFPE on line. 11(12),4817-4825. 10.5205/1981-8963-v11i12a15209p4817-4825-2017

Oliveira, M. L. C.D., Sousa; I. D. B. D., \& Arrais E. L. M. (2017). Prevenção de infecção Urinária: Indicadores de Qualidade da Assistência de Enfermagem em Idosos. J Nurs UFPE on line, 11(8), 3151-3157. 10.5205/reuol.11064-98681-4-ED.1108201721

Pereira, A. S. et al (2018). Metodologia da pesquisa científica. UAB/NTE/UFSM. https://www.ufsm.br/app/uploads/sites/358/2019/02/Metodologia-daPesquisa-Cientifica_final.pdf

Silva, D. R. M. (2019). Educação permanente em cateterismo vesical para prevenção de infecção do trato urinário. Reme - rev min enferm. (23)1 -9. $10.5935 / 1415-2762.20190067$

Sobreira, M. D. G. D. S. (2018). Prevenção de infecções na terapia intensiva: análise do conhecimento dos profissionais e construção de bundles. Universidade federal de campina grande - UFCG, http://dspace.sti.ufcg.edu.br:8080/jspui/handle/riufcg/8405.

Truzzi, J. C., Canalini A. F., Prezotti, J. A., \& Resplante, J. (2016). Recomendações SBU: cateterismo vesical intermitente. Sociedade Brasileira de Urologia. http://portaldaurologia.org.br/medicos/wp-content/uploads/2016/11/Recomendações_Cateterismo-Vesical-SBU-2016_final.pdf

Vidal, V. M., \& Gaite, B. F. (2018). Antisepsia en el sondaje urinario y en el mantenimiento de la sonda vesical. Med Intensiva. (43) S1,49-52. 10.1 016/j.medin.2018.09.014 